\title{
Social context modulates basic properties of oculomotor control
}

\author{
Alexander Strukelj \\ Centre for Languages and Literature, Lund University, Lund, Sweden \\ Tom Foulsham \\ Marcus Nyström \\ Department of Psychology, University of Essex, Colchester, Lund University Humanities Laboratory, Lund, Sweden. \\ UK.

\begin{abstract}
Experiments performed in a lab are often considered generalizable over both people and social settings. The problems with generalizing over different groups of people are well known, but it is only recently that changes in behavior depending on the social setting have been examined. Large changes in behavior can be seen in trivial cognitive tasks, depending on whether the participant is alone or if other people are present. However, there are very few studies which have measured eye movements in social settings. In this paper, we used the antisaccade task to test whether basic parameters of oculomotor control are sensitive to the size of an experimental group. Seventy participants conducted 48 antisaccade trials in groups of one to seven people in a classroom equipped with multiple eye trackers. The results show that for horizontal saccades, but not for vertical saccades, participants make significantly more antisaccade errors when the group size become larger. The group size did however not significantly predict a change in antisaccade latency. These results are in line with a number of recent studies on social attention showing that the mere presence of other people in the room can modulate several aspects of performance, and show that behavior in a lab might not be easily generalizable to everyday life or social situations. Finally, from a methodological viewpoint, the results show that the group size should be considered when testing participants in a social setting.
\end{abstract}

Keywords: social context, oculomotor control, antisaccade

\section{Introduction}

Our knowledge about eye movements and eye movement control is mainly acquired from laboratory-based experiments where participants are recorded individually in quiet rooms. While such experiments have provided substantial knowledge about the human visual system, it is unclear whether basic parameters of eye movements change when participants are tested in more natural situations such as classrooms, where other people are present. In other words, previous research is largely founded on the assumption that participants' behavior in simple saccade paradigms will be unaffected by the 'social context', which is defined as the influence of the "actual, imagined, or implied presence of

History: Received June 15, 2015; Published February 22, 2016

Citation: Strukelj, A., Foulsham, T., \& Nyström, M. (2016). Social context modulates basic properties of oculomotor control. Journal of Eye Movement Research, $9(2): 5,1 \sqrt{9}$

Digital Object Identifier: 10.16910/jemr.9.2.5

ISSN: 1995-8692

This article is licensed under a https://creativecommons.org/licenses/

by/4.0/Creative Commons Attribution 4.0 International license. others" Allport, 1968).

There are a number of reasons for doubting the assumption that eye movements would not change when other people are present. First, our attention and eye movements are affected by where other people are looking. For example, we often shift our attention in the direction of an eye gaze cue (e.g., Friesen \& Kingstone, 1998; Kuhn \& Kingstone, 2009). However, it has recently been acknowledged that when a gaze cue comes from a real person, as opposed to a picture of a person, behavioral and neurophysiological effects may be qualitatively different (Pönkänen, Peltola, \& Hietanen, 2011; Redcay et al. 2010; Risko, Laidlaw, Freeth, Foulsham, \& Kingstone 2012). In the particular case of gaze following, therefore, eye movements appear to be sensitive to the social context.

A second reason to investigate the influence of social context on basic eye movement behavior is that, where it has been examined, even the mere presence of another person can affect where people look. Laidlaw, Foulsham, Kuhn, and Kingstone (2011) compared participants' gaze behavior in a waiting room, both in the presence of a real person and when faced with a video of the same person. The results showed 
that the real person was looked at much less frequently than the video. The mere presence of another person can also change the target of gaze, even when the items being looked at are not themselves part of the social context. Richardson et al. (2012) reported that a person's gaze direction can be influenced merely by the knowledge that someone else is looking. Participants viewed a set of images, and they were sometimes told that another person was looking at the same images under the same conditions on the other side of the room. This knowledge altered which image was looked at most often. Importantly, Richardson et al. reported that shared exposure of a stimulus had to be accompanied with a shared task to produce the change in gaze direction.

Third, Strukelj, Brännström, Holmberg, Mossberg, and Holmqvist (in press) showed that antisaccades were affected by noise. Different sound presentations produced differences in error rates and latency. Notably, the only sounds that produced significantly higher saccadic error rates were social in nature, i.e., produced by humans. These were the sounds from children playing, the sounds from a crying baby, and non-distinct babble. Furthermore, music was found to significantly increase latency. This was interpreted as an implicit speed-accuracy tradeoff, making participants better at inhibiting reflexive eye movements at the cost of speed.

There is also evidence that the size of the group can modulate social context effects. For example, Gallup et al. (2012) investigated gaze cueing in the real world. Confederates looked up towards a camera in a busy street, and the researchers measured how this movement influenced passersby. A significant number of participants copied the looking behavior by following the gaze of the confederates. Importantly, the extent to which gaze-following occurred depended in a systematic way on the size of the group of gazing confederates. Gaze propagated more readily with larger stimulus groups, saturating at a group size of about 15 people. More generally, research on social context, including manipulations of the group size, has a long history in the social sciences (e.g., Zajonc, 1965; Bond \& Titus, 1983). Knowles (1983), for instance, found the students "learned more slowly, forgot less during rests, and recalled fewer peripheral aspects of the experiment" when the learning took place in front of a large audience $(N=8)$ compared to smaller audiences $(N=\{2,4\})$. Interestingly, also an implied social context such as wearing an eye-tracker (Risko \& Kingstone, 2011) or being video-taped (Miyazaki, 2015) can change participants' visual behavior. Risko and Kingstone (2011) reported that people wearing an eye-tracker are less likely to look at provocative stimuli, and argue that this reflects that the participants know that their eye movements are being monitored. However, the effect appears to last only for a certain period of time after which the participants resume their natural viewing behavior (Nasiopoulos, Risko, Foulsham, \& Kingstone, 2015). Along similar lines, Miyazaki
(2015) showed that video-taping participants improve their performance in a visual search task compared to when when the task is performed without video-taping.

The majority of previous work has focused on how gaze direction changes with social context. In this paper we address the more fundamental question of whether basic parameters of oculomotor control change when the social context is manipulated. The manipulation consisted of the number of participants being recorded simultaneously and in the same room while making antisaccades, i.e., inhibiting the reflex to launch a saccade toward an abruptly appearing target, and instead performing a saccade in the opposite direction. The antisaccade task is a standard test for control of saccadic eye movements and pre-frontal control, and is widely applied in neuropsychology (cf. Hutton \& Ettinger, 2006, for a review). Besides being a tool to assess brain dysfunction, antisaccades are sensitive to various cognitive components linked to working memory and goal activation. It is therefore a good task for investigating the cognitive component of social context.

Based on the previous literature on the influence of social context on gaze direction in addition to the observation that sounds of social nature influenced antisaccade performance (Strukelj et al., in press), we predicted that our minimal manipulation of social context-i.e., the number of participants simultaneously performing the task-would influence basic parameters of eye movement control as tested with the antisaccade task. It is however more difficult to predict the direction of the effects. A social facilitation, for instance, would mean that participants would try to perform better in the presence of others, which would be reflected in a lower antisaccade error rate and lower antisaccade latencies. On the other hand, the social context could require additional cognitive resources that would have the opposite effect and lead to poorer antisaccade performance. Since there are fundamental differences between horizontal and vertical saccades in many of their key features (Collewijn, Erkelens, \& Steinman 1988b, 1988a), and since previous work has shown that antisaccade metrics are sensitive to the position of the target, we ask participants to perform both horizontal and vertical saccades, and report the results separately (Dafoe, Armstrong, \& Munoz, 2007; Bonnet et al. 2013).

\section{Method}

\section{Participants}

A total of 70 native speakers of Swedish (47 female) between 18 and 33 years of age took part in the experiment. The participants were recruited through student lists and advertisements at Lund University. All participants had normal or corrected-to-normal vision. Due to technical problems with the recording and recording equipment, and to exclude participants with an insufficient number of accurate trials (see 


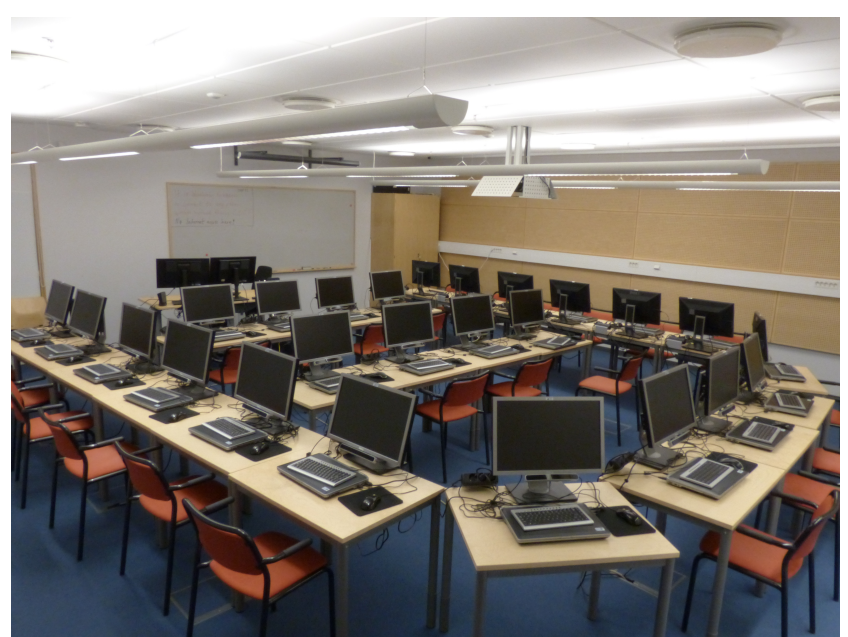

Figure 1. The Digital Classroom, Lund University, equipped with 25 RED-m eye trackers from SensoMotoric Instruments.

Data analysis-section), 14 participants were excluded from the results. This resulted in 56 (34 women) participants with a mean age of $22.4(S D=3.36)$ years. The participants were naïve to the purpose of the experiment. The experiments were carried out in accordance with the declaration of Helsinki.

\section{Apparatus}

Eye movement data were recorded at $120 \mathrm{~Hz}$ using RED$\mathrm{m}$ remote video-based eye trackers from SensoMotoric Instruments (Teltow, Germany). The recordings took place in the Digital Classroom at the Lund University Humanities Laboratory, Sweden. The classroom, depicted in Figure 1 . is equipped with 25 identical RED-m eye trackers. The distance from each participantâĂŹs eyes to the stimulus monitor was approximately $650 \mathrm{~mm}$, but varied somewhat for each participant and over the course of the experiment due to head movements. Stimuli were displayed on a Dell P2210 22 in. widescreen LCD display at a resolution of $1680 \times 1050$ pixels $(475 \times 300 \mathrm{~mm}$, equivalent to approximately $43.2 \times 28.1 \mathrm{de}-$ grees of visual angle) with a screen refresh rate of $60 \mathrm{~Hz}$. The eye tracker was controlled by SMI iView RED-m (v. 3.2.20), while stimulus presentation was handled by SMI Experiment Center 3.1.

\section{Stimuli}

Stimuli were presented in white color on a mid-gray background. For each trial, a fixation cross of size 0.65 degrees was presented in the center of the screen for a time $\Delta t_{1}$ randomly drawn from the interval $I \in U[1500,2000] \mathrm{ms}$. Directly after it disappeared, a circular target (diameter 0.54 degrees) appeared 12.5 degrees either to the left, right, up, or down, where it remained for another $\Delta t_{2}=1000 \mathrm{~ms}$, fol-

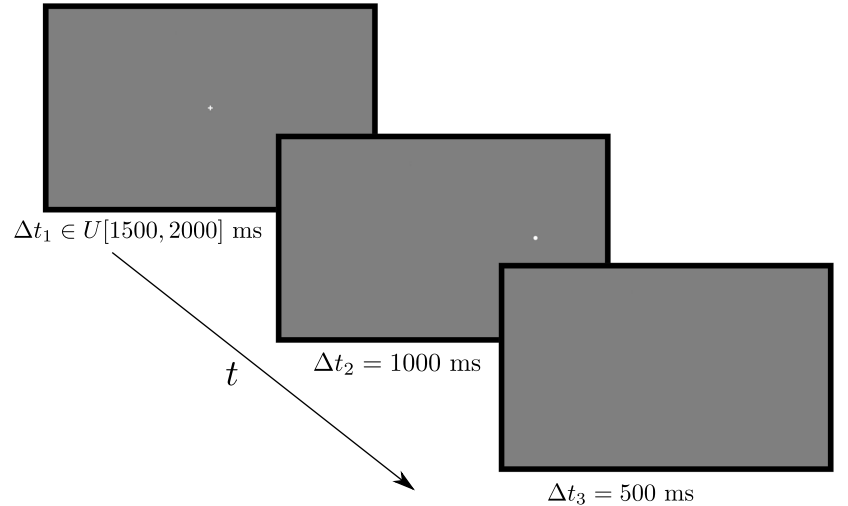

Figure 2. Time course of an antisaccade trial.

lowed by a blank screen present for $\Delta t_{3}=500 \mathrm{~ms}$. Following Strukelj et al. (in press), the current study consisted of 48 antisaccade trials, with 12 trials at each of the four target locations. The trial order was randomized. An example trial can be seen in Figure 2 .

\section{Procedure}

The experiment started with a 5 point calibration followed by a 4 point validation of the calibration accuracy. The average accuracy reported by Experiment Center was below one degree for all participants. During a trial, participants were instructed to fixate the central cross and then, as quickly and accurately as possible, perform a saccade in the opposite direction of the target, i.e., an antisaccade. This instruction was given orally to all participants during the introduction of the experiment, as well as in writing on the participants' screens directly before the task started.

Participants performed the task in groups of one to seven people (see Table 11). Ideally, the number of participants would be the same for each group size. In practice, this ideal sampling is difficult to obtain since some participants may not show up at all, or provide insufficient data quality due to a variety of reasons (see Nyström, Andersson, Holmqvist, \& van de Weijer, 2013, for examples).

When two or more participants were recorded, they were never seated directly next to each other or such that they could observe other participants' screens. They were aware of the fact that the other people in the room performed the same task, but proceeded through the experiment independently in the sense that each trial did not start at exactly the same time for all participants. Since the duration of the experiment was fixed, the participants also did not finish at exactly the same time. There was no explicit or implicit competition between participants.

Before and after the experiment was completed, the participants were engaged in other tasks unrelated to the experiment. They remained seated throughout all the tasks. Even though the participants were encouraged to sit as still as pos- 
Table 1

Total number of participants for each group size. Note that the number of participants is not always a multiple of the group size. This is due to the exclusion of participants with a poor data quality. The third column shows the number of recording sessions for each group size.

\begin{tabular}{ccc}
\hline Group size & \# participants & \# sessions \\
\hline 1 & 12 & 14 \\
2 & 8 & 4 \\
3 & 7 & 3 \\
4 & 14 & 4 \\
5 & 5 & 2 \\
6 & 5 & 1 \\
7 & 5 & 1 \\
\hline
\end{tabular}

sible in front of the eye tracker, visual and auditory disturbances reflected a normal classroom group setting, e.g., adjusting one's position in the chair, coughing, or asking for assistance from the experimenter. Two experiment leaders were present in the room at the time of the recording to help people with issues related to obtaining a proper seating position, as well as an acceptable calibration.

\section{Data analysis}

Raw eye movement data were exported using BeGaze 3.4. The data processing, statistical analyses, and plots were conducted with Matlab R2015a (Natick, Massachusetts) and $\mathrm{R}$ (R Core Team, 2015, v. 3.2.0). Saccades were detected with the microsaccade algorithm by Engbert and Kliegl (2003), using a minimum saccade duration of four samples (33.3 ms) and a $\lambda=4$, which controls the threshold used to separate samples with high velocity, i.e., the saccades, from those with low velocity. Since microsaccades and saccades share many dynamic properties, Engbert and Kliegl's algorithm also work well on larger, voluntary saccades. Saccade latency was defined as the time from the appearance of the target until the onset of a saccade was detected.

Following the standardized analysis protocol suggested by Antoniades et al. (2013), each antisaccade trial was classified as either a correct antisaccade, an incorrect antisaccade, a non-response, or a mis-recording. In the correct trials, the first saccade after trial onset was launched within $\pi / 4 \mathrm{rad}$ of the direction opposite the target. An incorrect antisaccade occurred when the initial saccade was launched in any other direction than the correct one. Non-responses occurred when no saccade was detected during a trial. A trial was considered a mis-recording when any of the following criteria was fulfilled: 1) the initial fixation location was more than two degrees away from the central fixation cross at the onset of a trial. 2) There were more than $50 \%$ lost samples in the trial. In the RED-m data files, this means that $(x=0, y=0)$-coordinates were present. Besides the nonresponses, only trials with a saccade latency larger than 50 ms, a saccade peak velocity less than $1000 \mathrm{deg} / \mathrm{s}$, a saccade amplitude larger than one degree, and those that were not classified as mis-recordings, were considered in the analysis. Horizontal and vertical antisaccades were analyzed separately. Participants who had fewer than five $(21 \%)$ valid antisaccade trials in a certain direction, for whatever reason, were excluded.

\section{Results}

We used conservative criteria to exclude any trials where data were lost due to participant movement or equipment issues. For horizontal saccades, these criteria resulted in an average of $20 \%$ of trials being classed as mis-recordings, a proportion which did not differ significantly according to group size $(\mathrm{r}=0.16, p=.25)$. For vertical saccades this number changed to $21 \%(\mathrm{r}=0.32, p=.02)$. Of the remaining valid trials for horizontal saccades, $61 \%$ (vertical saccades $56 \%$ ) were correctly performed antisaccades, while $28 \%$ (vertical saccades $34 \%$ ) were incorrect saccades (normally towards the target) and the remaining $11 \%$ (vertical saccades $10 \%$ ) were non-responses. Incorrect antisaccades and non-responses were merged into a single class called antisaccade errors 11 Excluding the mis-recordings, the proportion of errors therefore equals one minus the proportion of correctly performed antisaccades.

Figures 3 and 4 illustrate how antisaccade errors and latency are affected by the group size for horizontal (Figure 3 and vertical (Figure 4) saccades. The figures in the left columns show the proportion of antisaccade errors and the right figures latencies for correctly performed antisaccades. Each data point represents one participant and the lines illustrate least square fits of the data.

Considering only the group size with a single participant $(N=12)$, the proportion of errors is $32 \%$ for horizontal saccades and $41 \%$ for vertical saccades. This is slightly higher than what has previously been reported for healthy participants; in their review on antisaccades, Hutton and Ettinger (2006) refer to studies that report error rates between 5 and $25 \%$, but also emphasize that the results differ significantly across studies and laboratories. It should be noted that the antisaccades in this paper were performed with relatively few trials, potentially explaining the slightly higher than usual error rates. For the largest group sizes including six or seven people the performance has dropped significantly and is not better than chance, irrespective of the target direction.

In the typical situation with only one participant, the latency is just about $300 \mathrm{~ms}$, and does not change notable due to the saccade direction. Latencies of similar magnitude have

\footnotetext{
${ }^{1}$ Whether the non-responses were included or not did not change the results.
} 

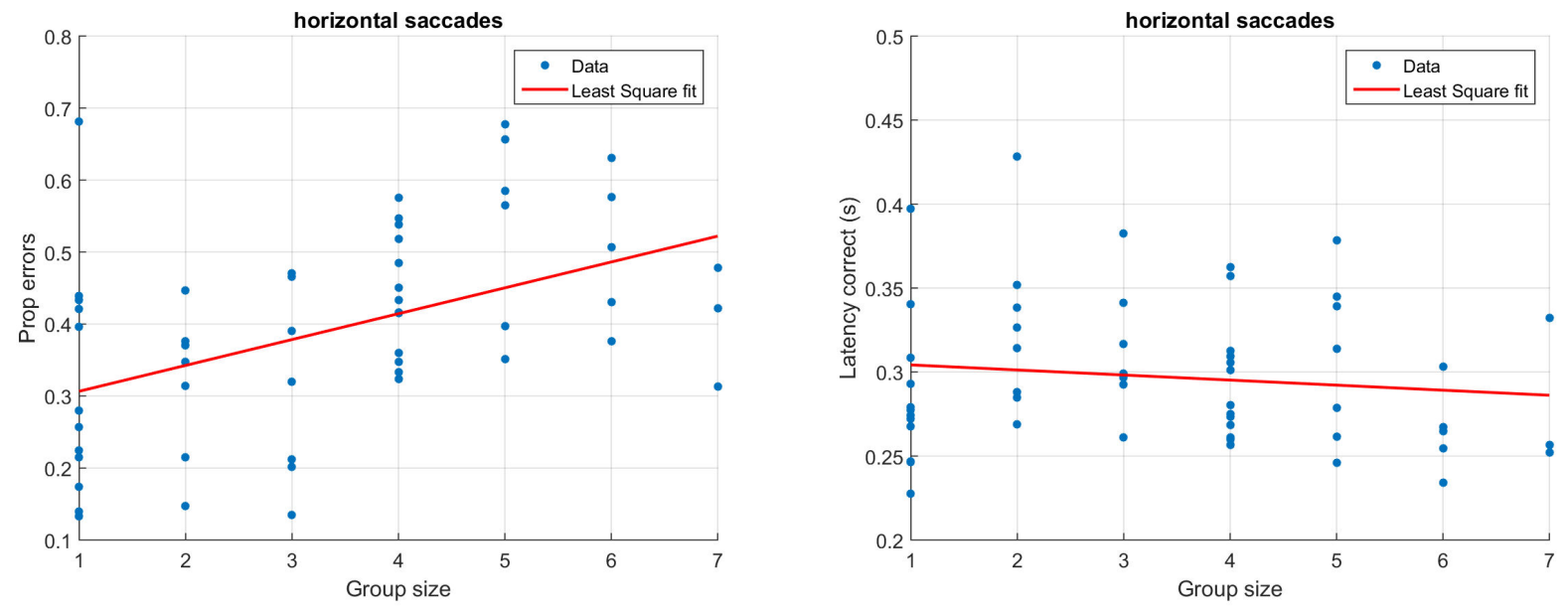

Figure 3. Proportion of antisaccade errors (left) and latency of correct antisaccades (right) for horizontal saccades. Each dot represents average results from one participant.
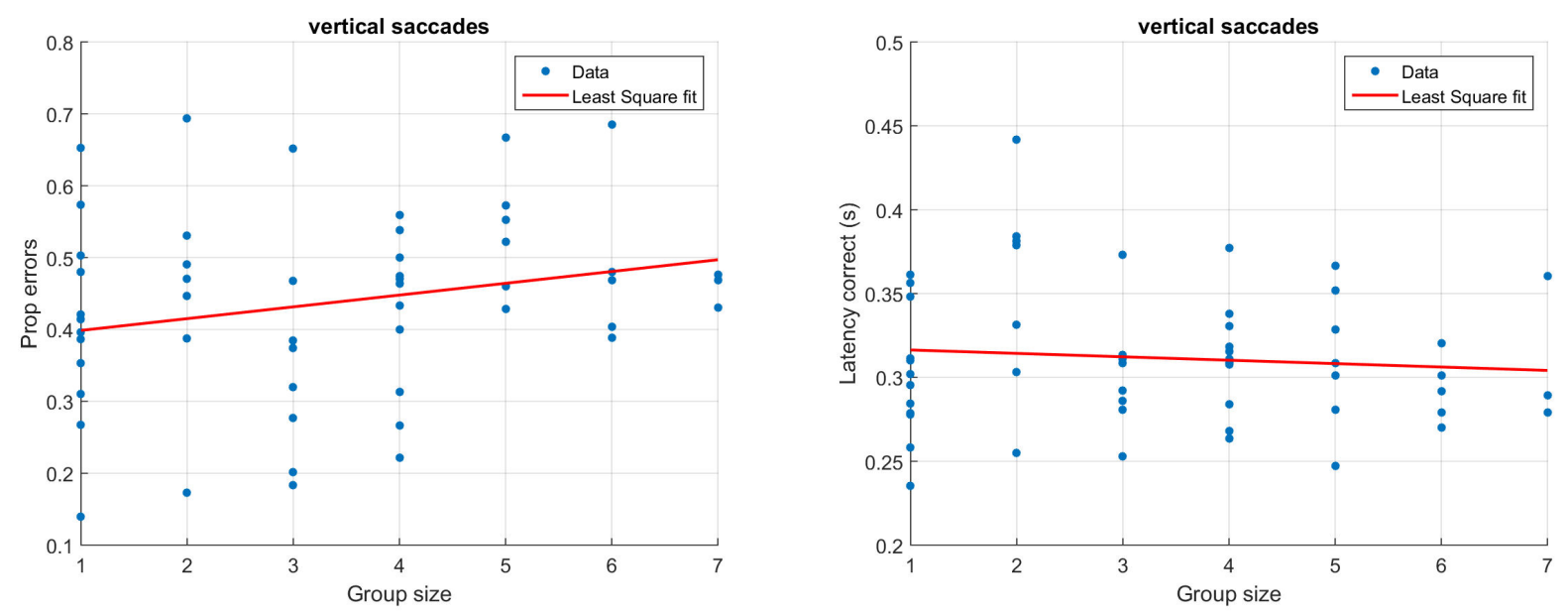

Figure 4. Proportion of antisaccade errors (left) and latency of correct antisaccades (right) for vertical saccades. Each dot represents average results from one participant.

been reported previously (e.g., Butler, Zacks, \& Henderson, 1999, Petrovsky et al. 2009). As illustrated in Figures 3 and 4 (right), participants being recorded in larger groups produce slightly shorter saccade latencies. Despite the relatively low number of trials, typical results such as a higher latency for correct $(M=302, S D=39 \mathrm{~ms})$ compared to incorrect $(M=257, S D=79 \mathrm{~ms})$ trials could be replicated in the single participant group using only horizontal saccades $(t(1)=2.28, p=0.03$, paired $t$-test) (Everling, Spantekow, Krappmann, \& Flohr, 1998, Munoz \& Everling 2004 Jazbec, McClure, Hardin, Pine, \& Ernst, 2005). To quantify the above observations statistically, linear models were fit to the data using group size to predict the proportion of errors as well as latencies. Separate models were fit depending on the direction of the target. For horizontal sac- cades, the group size significantly predicted the proportion of errors $\left(\beta=0.04, t(54)=3.88, p<0.001, R^{2}=0.20\right)$. The estimate $\beta$ indicates that for each additional participant that is present during the recording, there is a 0.04 increase in the proportion of errors. The group size has a smaller and only marginally significant on vertical errors $(\beta=0.02$, $\left.t(52)=1.69, p=0.09, R^{2}=0.03\right)$. To explicitly test whether the saccade direction predicts the proportion of errors, we ran an additional linear model including both group size and saccade direction. This model showed that there was an overall effect of both group size $(\beta=0.04, t(106)=3.80, p<0.001)$ and saccade direction $(\beta=0.11, t(106)=2.15, p=0.03)$, but no significant interaction between them $(\beta=-0.02$, $t(106)=-1.46, p=0.15), R^{2}=0.20$. With regards to latencies, the effect of group size was larger but non-significant 
for horizontal saccades $(\beta=-0.003, t(54)=-.93, p=0.36$, $\left.R^{2}=-0.002\right)$ compared to vertical saccades $(\beta=-0.002$, $\left.t(52)=-0.66, p=0.52, R^{2}=-0.01\right)$. It should be noted that a linear model is adequate only for reasonably small group sizes; for larger group sizes, the model would predict an unreasonably high error rate.

\section{Discussion}

Although millions of antisaccades have been recorded in labs around the world (Antoniades et al., 2013), we do not know how such tasks vary with social context. The results showed that, rather than being a simple, repeatable, and stereotyped response, antisaccade performance changes according to the number of people simultaneously performing the task in the same room. More people, i.e., a stronger social context, leads to worse performance. There was also some evidence that the magnitude of the change depends on whether the saccades are performed in the horizontal or the vertical direction, where the latter direction is less sensitive to the size of the group being tested. This has obvious implications for those of us investigating attention and inhibition in crowded settings such as a classroom or a busy clinic. It also adds to previous research demonstrating that the mere presence or implied presence of other people - a minimal social context-can affect how people distribute their attention (Richardson et al., 2012, Tufft, Gobel, \& Richardson, 2015).

Antisaccade performance depends on several factors such as age (Butler et al. 1999), mental workload (Roberts, Hager, \& Heron 1994), training (Dyckman \& McDowell, 2005), and differences in task instructions (Taylor \& Hutton, 2009), as well as being disrupted in certain patient groups (see overview by Hutton \& Ettinger, 2006). The Digital Classroom aims to emulate an environment that resembles a school classroom. This allows for testing to be conducted in situations that more closely resemble real life. Perhaps the most accessible explanation for the findings in this paper would be that the added presence of others affects the physical environment in the classroom, such that participant-induced disturbances draw attention away from the experimental task. The nature of such disturbances could be both auditory (e.g., someone coughing or sighing) and/or visual (e.g., head or hand movement). For instance, it has been shown that distraction in terms of auditory stimulation affect saccade latencies (Halliday \& Carpenter, 2010), and that certain types of sounds produce different antisaccade performance compared to conducting antisaccades in silence (Strukelj et al. in press). Interestingly, in Strukelj et al. all sounds that increased error rates involved sounds from a social context, namely children playing, a crying baby, and babble noise. Such distraction may also account for the rather high proportion of mis-recordings that was observed.
However, while the results could be interpreted as arising solely from noise disturbance in the physical environment, we believe this not to be the case. The room was relatively silent during recording, and any disturbance would be much reduced compared to experiments which have played disturbing sounds directly to a participant (Halliday \& Carpenter. 2010, Strukelj et al. in press). Furthermore, visual disturbance was held at a minimum, as all participants performed a task on the computer both prior to the antisaccade task and after its completion. Thus, no participant moved away from a computer during the recording of the task.

An alternative possibility is that the decreased antisaccade performance in larger groups is a more general effect of the presence of others. There is a large literature on âAJJmere presenceâĂİ effects, most of which focuses on improvements in performance with larger groups (social facilitation; see Guerin, 1986 for a review). In fact, the presence of others tends to increase performance in easy or well-practiced tasks but decrease performance in complex or difficult tasks ( $\mathrm{Za}$ jonc, 1965; Bond \& Titus, 1983 ). To the extent that the antisaccade task is a difficult one, therefore, our results fit with previous literature using non-eye movement dependent measures. By this account, participantsâĂŹ errors increased in the presence of others because of an increase in arousal due to social monitoring and awareness that they might be evaluated by others (even though this was not the case). Such effects have not previously been demonstrated in an eye movement task.

It is important to note that performance differences in the present experiment might have affected both error rate and saccade latency. There is some evidence that complex tasks are performed slower, as well as less accurately, in the presence of a group (Bond \& Titus, 1983). This was not the case in the present study, where latency showed only a marginal change in larger groups (and one indicating that participants actually got slightly quicker). The results may therefore reflect a speed-accuracy tradeoff which increases with group size. While the non-significant decrease in latency speaks against this explanation, it may be that the effect has saturated. Several studies report antisaccade latencies slightly above $300 \mathrm{~ms}$ for healthy participants recorded individually (e.g., Butler et al., 1999; Petrovsky et al., 2009). Thus, it may be difficult to decrease the latency even further irrespective of the type of task or manipulation. The presence of additional people in the room may have encouraged participants to try to respond faster, leading to increased errors.

Previous research has observed increased errors in the antisaccade task with distraction or cognitive load (Halliday \& Carpenter, 2010; Stuyven, Van der Goten, Vandierendonck, Claeys, \& Crevits, 2000), but the reported effect of such manipulations on latency varies. In a go/no-go task, Halliday and Carpenter (2010) observed more low latency saccades, but no overall change in average latency, with increased au- 
ditory distraction. Stuyven et al. (2000), in contrast, found longer average anti-saccade latencies with a concurrent distraction task. Taylor and Hutton (2009) found that asking participants to react more quickly did reduce antisaccade latencies, but had no knock-on effect on accuracy. Thus, evidence of a speed-accuracy tradeoff in antisaccades is mixed. Such a tradeoff might indeed be encouraged by social facilitation, for example when workers are being monitored (Brewer \& Ridgway, 1998). It is important to note that, because participants carried out further tasks following the antisaccade trials, finishing quickly did not provide a clear communication to other group members.

Although participants were not tested in complete synchrony, they knew that others were engaged in the same task. In the experiments by Richardson et al. (2012), the knowledge that someone else was looking at the same image, and for the same reason, made participants look more towards negatively-valenced items in the display. One of the explanations proposed for this finding is that participants in a group are trying to âĂŸalignâĂŹ with other group members, a process which amplifies a bias towards negative items. In the present experiment, it is conceivable that this attempt at alignment interfered with the ability to inhibit a saccade towards the target, which is a difficult task. This will be important to test in further research by manipulating the task and the coordination between group members. For example, would we get different results if all group members were performing a pro-saccade task, or if task varied between group members?

The introduced social context may affect horizontal and vertical antisaccades differently, and the effect on performance was significant only when antisaccades were in the horizontal direction. There are at least two explanations to why this result was obtained. First, one might argue that the additional social context was horizontal in nature since the participants sat next to-and not above or below-each other. To further probe this spatial aspect of the social context, one could create conditions using seating positions with people present on both sides of a participant, only to the right, or only to the left. Unfortunately, the positions of the participants in the room were not recorded in the current experiment. However, no participants were ever seated next to each other, and no participant saw other screens apart from their own. Second, horizontal and vertical saccades are considerably different in many of their main descriptive features, e.g., peak velocity and accuracy, and may be generated by systems with different properties (Collewijn et al. 1988b. 1988a). For instance, vertical saccades are less accurate than horizontal saccades (Collewijn et al. 1988b), and also show up-down asymmetries in terms of e.g., peak velocity (Becker \& Jürgens, 1990). Horizontal and vertical saccades are also represented differently in the superior colliculus (SC) (Hall \& Moschovakis, 2003; Van der Stigchel \& Theeuwes, 2008).
It is possible that each subsystem is affected differently by the manipulated social context, and that the degree of social context modulates the response. There is evidence from functional magnetic resonance imaging (fMRI), for instance, that the activity in the SC was reduced in response to a threat when holding hands with the spouse compared to an anonymous experimenter (Coan, Schaefer, \& Davidson, 2006).

While one could speculate about why horizontal and vertical saccades are affected differently by the social context, the differences we observe are small and need to be validated by future studies.

It should be noted that even the single group size could be considered a social context since two operators were present during the recordings. Even though operators are present during many 'traditional' eye-tracking experiments, further experiments are required to quantify the effect of their presence.

As experimental hardware such as eye-tracking equipment becomes cheaper and more widely available, there will be increasing opportunities for testing larger groups. While this offers advantages in terms of time and effort, it may also give us new avenues for investigating the role of social context. These investigations have the potential to reveal a lot about even simple cognitive tasks. However, while a facility with multiple eye trackers offers the possibility to increase the throughput of participants, it also calls for a critical view of the methods and results, as the results may vary as a function of the number of participants being recorded.

There are several unexplored aspects of how the presence of others affects people's basic eye movement control and gaze behavior in a multi-eye-tracker setup. Future work includes quantifying the relative contribution of bottom-up factors originating from changes in the physical environment and top-down factors originating from participantsâĂŹ knowledge that other people are present and are performing the same or other tasks.

\section{Acknowledgments}

We gratefully acknowledge and thank Manuel Oliva for help with the data collection. We also acknowledge the support from the Lund University Humanities Laboratory, where the recordings were performed.

\section{References}

Allport, G. (1968). The historical background of modern social psychology. In The handbook of social psychology (pp. 1-80). Addison-Wesley Reading, MA.

Antoniades, C., Ettinger, U., Gaymard, B., Gilchrist, I., Kristjánsson, A., Kennard, C., ... Carpenter, R. H. (2013). An internationally standardised antisaccade protocol. Vision Research, 84, 1-5. 
Becker, W., \& Jürgens, R. (1990). Human oblique saccades: quantitative analysis of the relation between horizontal and vertical components. Vision Research, 30(6), 893920.

Bond, C. F., \& Titus, L. J. (1983). Social facilitation: a metaanalysis of 241 studies. Psychological Bulletin, 94(2), 265.

Bonnet, C., Hanuška, J., Rusz, J., Rivaud-Péchoux, S., Sieger, T., Majerová, V., ... Ružička, E. (2013). Horizontal and vertical eye movement metrics: What is important? Clinical Neurophysiology, 124(11), 22162229.

Butler, K. M., Zacks, R. T., \& Henderson, J. M. (1999). Suppression of reflexive saccades in younger and older adults: Age comparisons on an antisaccade task. Memory $\mathcal{E}$ Cognition, 27(4), 584-591.

Coan, J. A., Schaefer, H. S., \& Davidson, R. J. (2006). Lending a hand social regulation of the neural response to threat. Psychological Science, 17(12), 1032-1039.

Collewijn, H., Erkelens, C. J., \& Steinman, R. (1988a). Binocular co-ordination of human horizontal saccadic eye movements. The Journal of Physiology, 404(1), 157-182.

Collewijn, H., Erkelens, C. J., \& Steinman, R. (1988b). Binocular co-ordination of human vertical saccadic eye movements. The Journal of Physiology, 404(1), 183-197.

Dafoe, J. M., Armstrong, I. T., \& Munoz, D. P. (2007). The influence of stimulus direction and eccentricity on proand anti-saccades in humans. Experimental Brain Research, 179(4), 563-570.

Dyckman, K. A., \& McDowell, J. E. (2005). Behavioral plasticity of antisaccade performance following daily practice. Experimental Brain Research, 162(1), 6369.

Engbert, R., \& Kliegl, R. (2003). Microsaccades uncover the orientation of covert attention. Vision Research, 43(9), 1035-1045.

Everling, S., Spantekow, A., Krappmann, P., \& Flohr, H. (1998). Event-related potentials associated with correct and incorrect responses in a cued antisaccade task. Experimental Brain Research, 118(1), 27-34.

Friesen, C. K., \& Kingstone, A. (1998). The eyes have it! reflexive orienting is triggered by nonpredictive gaze. Psychonomic Bulletin E Review, 5(3), 490-495.

Gallup, A. C., Hale, J. J., Sumpter, D. J., Garnier, S., Kacelnik, A., Krebs, J. R., \& Couzin, I. D. (2012). Visual attention and the acquisition of information in human crowds. Proceedings of the National Academy of Sciences, 109(19), 7245-7250.

Hall, W. C., \& Moschovakis, A. K. (2003). The superior colliculus: new approaches for studying sensorimotor integration. CRC Press.
Halliday, J., \& Carpenter, R. H. (2010). The effect of cognitive distraction on saccadic latency. Perception, 39(1), 41.

Hutton, S. B., \& Ettinger, U. (2006). The antisaccade task as a research tool in psychopathology: A critical review. Psychophysiology, 43(3), 302-313.

Jazbec, S., McClure, E., Hardin, M., Pine, D. S., \& Ernst, M. (2005). Cognitive control under contingencies in anxious and depressed adolescents: an antisaccade task. Biological Psychiatry, 58(8), 632-639.

Knowles, E. S. (1983). Social physics and the effects of others: Tests of the effects of audience size and distance on social judgments and behavior. Journal of Personality and Social Psychology, 45(6), 1263-1279.

Kuhn, G., \& Kingstone, A. (2009). Look away! eyes and arrows engage oculomotor responses automatically. Attention, Perception, E Psychophysics, 71(2), 314-327.

Laidlaw, K. E., Foulsham, T., Kuhn, G., \& Kingstone, A. (2011). Potential social interactions are important to social attention. Proceedings of the National Academy of Sciences, 108(14), 5548-5553.

Miyazaki, Y. (2015). Influence of being videotaped on the prevalence effect during visual search. Frontiers in Psychology, 6, 583.

Munoz, D. P., \& Everling, S. (2004). Look away: the antisaccade task and the voluntary control of eye movement. Nature Reviews Neuroscience, 5(3), 218-228.

Nasiopoulos, E., Risko, E. F., Foulsham, T., \& Kingstone, A. (2015). Wearable computing: Will it make people prosocial? British Journal of Psychology, 106(2), 209-216.

Nyström, M., Andersson, R., Holmqvist, K., \& van de Weijer, J. (2013). The influence of calibration method and eye physiology on eyetracking data quality. Behavior Research Methods, 45(1), 272-288.

Petrovsky, N., Weiss-Motz, F., Schulze-Rauschenbach, S., Lemke, M., Hornung, P., Ruhrmann, S., ... Wagner, M. (2009). Antisaccade performance is related to genetic loading for schizophrenia. Journal of Psychiatric Research, 43(3), 291-297.

Pönkänen, L. M., Peltola, M. J., \& Hietanen, J. K. (2011). The observer observed: Frontal EEG asymmetry and autonomic responses differentiate between another person's direct and averted gaze when the face is seen live. International Journal of Psychophysiology, 82(2), 180-187.

R Core Team. (2015). R: A language and environment for statistical computing [Computer software manual]. Vienna, Austria. Retrieved from http://wwW .R-project.org/

Redcay, E., Dodell-Feder, D., Pearrow, M. J., Mavros, P. L., Kleiner, M., Gabrieli, J. D., \& Saxe, R. (2010). Live 
face-to-face interaction during FMRI: A new tool for social cognitive neuroscience. Neuroimage, 50(4), 1639-1647.

Richardson, D. C., Street, C. N., Tan, J. Y., Kirkham, N. Z., Hoover, M. A., \& Cavanaugh, A. G. (2012). Joint perception: Gaze and social context. Frontiers in Human Neuroscience, 6, 1-8.

Risko, E. F., \& Kingstone, A. (2011). Eyes wide shut: implied social presence, eye tracking and attention. Attention, Perception, $\mathcal{E}$ Psychophysics, 73(2), 291296.

Risko, E. F., Laidlaw, K. E., Freeth, M., Foulsham, T., \& Kingstone, A. (2012). Social attention with real versus reel stimuli: Toward an empirical approach to concerns about ecological validity. Frontiers in Human Neuroscience, 6, 1-11.

Roberts, R. J., Hager, L. D., \& Heron, C. (1994). Prefrontal cognitive processes: Working memory and inhibition in the antisaccade task. Journal of Experimental Psychology: General, 123(4), 374.

Strukelj, A., Brännström, J., Holmberg, N., Mossberg, F., \&
Holmqvist, K. (in press). The impact of sound presentations on executive control: Evidence from eye movements. Psychology of Music.

Taylor, A. J., \& Hutton, S. B. (2009). The effects of task instructions on pro and antisaccade performance. $E x$ perimental Brain Research, 195(1), 5-14.

Tufft, M., Gobel, M., \& Richardson, D. C. (2015). Social eye cue: How knowledge of another personâĂŹs attention changes your own. In D. C. Noelle, W. A. S. Dale R., M. T. Yoshimi J., C. D. Jennings, \& P. P. Maglio (Eds.), Proceedings of the 37th annual meeting of the cognitive science society. Austin, TX: Cognitive Science Society.

Van der Stigchel, S., \& Theeuwes, J. (2008). Differences in distractor-induced deviation between horizontal and vertical saccade trajectories. Neuroreport, 19(2), 251254.

Zajonc, R. B. (1965). Social facilitation. Science, 149, 269 274. 